\title{
DIE NERONISCHEN WANDMALEREIEN IN DER VILLA DER POPPAEA VON OPLONTIS
}

\author{
BERNARD ANDREAE
}

Folgende Tatsachen sprechen dafür, daß die große, 1968 entdeckte Villa mitten im Stadtgebiet von Torre Annunziata, 4 km nördlich vor den Toren Pompejis an einem Ort, der auf der Tabula Peutingeriana die Bezeichnung Oplontis trägt, Poppaea, der Frau Neros gehörte und aus ihrem Besitz in den des Kaisers übergegangen ist:

1. In der Villa wurde eine Amphora mit der rot aufgemalten Adresse: SECUNDO PoppaAe, dem Secundus, Freigelassenen der Poppaea, gefunden.

2. Ein Graffito auf der im Architekturstil bemalten Wand des Domestikentraktes lautet: MNESTE BERYLLOS, das heißt „Beryllos denk’ daran!“ Der Spottname Beryllos dürfte der Beryll gewesen sein, von dem das deutsche Wort Brille abgeleitet ist, ein Halbedelstein, den der fehlsichtige Nero als Vergrößerungsglas benutzte.

3. Die Villa, die um die Mitte des 1. Jahrhunderts v. Chr., als Mitglieder der Familie der Secundii in Rom hohe Ämter innehatten, ungemein aufwendig erbaut und im Architekturstil ausgemalt worden war, wurde bei einem Erdbeben $62 \mathrm{n}$. Chr. beschädigt. Man begann sofort mit der Wiederherstellung, und vor allem legte man jenseits des Domestikentraktes im Süden einen neuen große Flügel der Villa an mit dem größten aus den Vesuvstädten bekannten Schwimmbecken. Außerdem wurde ein großer, langgestreckter Wartesaal für die Klientel mit einfachen aufgemauerten Bänken für an die hundert Personen sowie eine Art Audienzsaal in Form eines Fünfachtelschlusses erbaut. Die Ausbesserungs- und Erweiterungsarbeiten wurden aber kurze Zeit darauf, spätestens nach zwei Jahren eingestellt; alles wurde unvollendet liegengelassen. Das dürfte wohl im Jahre $65 \mathrm{n}$. Chr. gewesen sein, als die schwangere Poppaea, die Nero vor den Leib getreten hatte, vorzeitig niederkam und starb. Danach war die riesige Villa bis zum Vesuvausbruch vierzehn Jahre später, im Herbst $79 \mathrm{n}$. Chr., unbewohnt und unbenutzt. Man hat in der Villa, die durch den Vesuvausbruch versiegelt wurde, keinerlei Mobiliar festgestellt wie sonst in dem unter ähnlichen Bedingungen verschütteten Herculanum; die Küche war 
unbenutzt, die Latrine mit leeren Amphoren vollgestellt, und bei den Ausgrabungen wurde eine einzige Leiche gefunden, wohl der Wächter des Hauses, das offenbar in kaiserlichen Besitz übergegangen war. Verständlicherweise wollte Nero dort nicht mehr wohnen, und auch seine flavischen Nachfolger scheinen kein Interesse daran gehabt zu haben. Wäre die Villa in Privatbesitz geblieben, wäre eine über so lange Zeit nicht erfolgte Nutzung in keiner Weise zu erklären. Die Ausmaße der Villa und besonders des neu geplanten und auf die Bedürfnisse eines amtierenden Kaisers zugeschnittenen Traktes sind enorm.

Der Stil der Wandmalereien lehrt, daß die Villa um 50 v. Chr. in Betrieb genommen wurde und die Abfolge der darin anzutreffenden Malstile zeigt, daß sie ständig verändert wurde. Man kann an diesen Stilen die ganze Entwicklung der römisch-kampanischen Wandmalerei vom sogenannten zweiten, bis zum vierten Stil ablesen. Die Forschung hat in der Zwischenzeit gezeigt, daß die einfache Einteilung der Malerei in vier Stile von August Mau aus der Zeit um 1880 eine kontinuierliche Entwicklung der illusionistischen Architekturmalerie spätrepublikanischer Zeit, über den Flächen- und Kandelaberstil julisch-claudischer Zeit zu einem unter Nero einsetzenden Phantasiestil darstellt, der in flavischer Zeit seinen Höhepunkt erreicht, aber auch zu erstarren beginnt.

Die Villa bietet in ihren zahlreichen Räumen eine vollständige Abfolge dieser Stile, so daß es möglich ist, die chronologische Reihenfolge der Ausmalung zu bestimmen: Man begann mit dem Atrium, das die größten aus dem Altertum erhaltenen, bemalten Wände zeigt. Das makedonische Palastarchitektur illusionistisch nachahmende Wandsystem ist noch geschlossen. Auf dem Wandputz, unter der Malschicht, wurde aber nicht nur die rot aufgeschnürte Quadratur für die Proportionierung der Wandmalerei gefunden, sondern auch eine Entwurfsskizze für das Wandsystem des daneben liegenden Tricliniums, das heißt des Speiseraums der Villa. Dieses aufwendige und besonders reiche Wandsystem zeigt schon die typische, achsialsymmetrische, illusionistische Öffnung der Wände mit Ausblick durch eine Mittelädicula und mit seitlichen Bogenöffnungen der Flügel. Berühmt sind die als Trompe-l'œil gestalteten Gegenstände, zum Beispiel der Feigenkorb. Vergleicht man diesen aus Weidenruten geflochtenen Korb mit einem ähnlichen Korb aus dem Ixionzimmer des frühflavischen Hauses der Vettier in Pompeji, so erkennt man, daß sich die Maltechnik auch stilistisch in Richtung auf eine optische Form verändert, um den von Alois Riegl begrifflich erfaßten Gegensatz von haptischer 
und optischer Kunststruktur aufzunehmen. Die weitere Abfolge der Ausmalung der einzelnen Räume führt über die große scenae frons des anschließenden Saales (großer oecus) mit den über die Balustrade balanzierenden Pfauen, die mit den Pfauen neronischer Zeit zu vergleichen sind, um die Stilentwicklung zu erkennen, weiter über die Scheinarchitektur des Cubiculums zum Maskenzimmer mit seiner dionysischen Bilderwelt und dem berühmten Korb mit dem Gazeschleier. Bis hierhin reicht der Architekturstil der vierziger Jahre des 1 . Jahrhunderts v. Chr.

In den zwanziger Jahre, also in augusteischer Zeit, wurden das Calidarium der Thermen, ein Saal mit tubulierten Wänden und Hypokausten in einem an die Villa Imperiale in Pompeji anschließenden Stil ausgemalt. Man kann hier verfolgen, wie der Durchblick durch die Mittelädikulen der Hauptwände zum Anblick wird und ein solcher an die Stelle des Durchblicks treten kann, wie es als datiertes Beispiel die Villa unter der Farnesina in Rom bietet. Der jenseits der Wand liegende imaginäre Raum fällt als Bild in die Ebene der Wand, und die als vor der Wand gedachten, gemalten Architekurelemente, Säulen und Gebälke, ziehen sich wie Kandelaber und Trennungsstreifen in die Ebene der Wand zurück, die dadurch als Malfläche erwiesen wird und alsbald auch mit phantastischen Architekturen bemalt werden kann. Die Decke und der obere Teil des Thermensaales wurde später, wohl in der Zeit des Kaisers Claudius erneuert. Hier findet man auch Darstellungen von Pfauen, die zum neronischen Stil der Villa überleiten. Aus spätclaudischer Zeit dürfte das flächig bemalte, rote Cubiculum am Rande zum Domestikentrakt stammen.

Unser eigentliches Interesse gilt den interessanten Wandmalereien im neronischen, zwischen 63 und $65 \mathrm{n}$. Chr. ausgemalten, aber nicht fertig gewordenen Südtrakt der Villa und besonders dem zentralen Speisesaal, der mit einem schönen Inarsienfußboden aus Buntmarmoren geschmückt ist und eine außerordentlich raumaufwendige archtitektonische Ausgestaltung zeigt. Die Anlage der Räume und die Wandmalerei bilden hier ein ineinandergreifendes Ganzes. Zu beiden Seiten des rechteckigen Tricliniums liegt eine symmetrische Abfolge von Räumen, durch die man in einer von Wandöffnungen ermöglichten Blickachse über abwechselnd offene, also belichtete, schachtartige Höfe und dunkle kleinere, zum Schwimmbecken hin offene Zimmer hindurch blicken kann. Die gerahmten Durchblicke pompejanischer Gartenarchitekturen werden hier durch bemalte Wände mit Fensteröffnungen von der Zweidimensionalität in die Dreidimensionalität übergeführt. Die Fensteröffnungen werden von innen nach außen immer kleiner, so daß hintereinander gestaffelte, 
ineinander geschachtelte, Hell- und Dunkelzonen entstehen, die durch pervertierte Gartenmalereien auf gelbem Grund bereichert sind, um den Titel des Kolloquiums aufzugreifen.

Die Entwicklung des Typus der römischen Gartenmalereien kann man parallel zur Ausmalung der Villa von Oplontis seit dem Gartensaal der Livia aus ihrer Villa frühaugusteischer Zeit bei Primaporta über kontinuierliche Beispiele aus Pompeji bis in neronische Zeit verfolgen, als die Villa von Oplontis in dieser höchst phantasievollen Weise ausgemalt wurde. Die ,Pervertierung ‘ erfolgte auf der ganzen Linie, nicht nur werden die Grenzen von Architektur und Malerei aufgehoben, sondern auch Natur und Kunst greifen ineinander. Der Boden der schachtartigen Höfe, in die der Blick aus dem Triclinium nach beiden Seiten geht, war bepflanzt und deren Wände wurden mit Pflanzen bemalt. Doch der Grund ist nicht blau wie der Luftraum hinter den Pflanzen und Vögeln der Villa der Livia bis hin zur flavischen Casa della Venere in Conchiglia in Pompeji, sondern er ist in völlig unnatürlicher Weise gelb. Die Pflanzen und Vögel sind auch nicht naturalistisch gemalt wie in Primaporta, sondern in einem ganz eigenen Stil, für den eine treffende, begriffliche Bezeichnung noch zu finden ist. Man kann diesen Stil bei einem Vergleich der Pfauen von der scenae frons des großen oecus, das heißt des Saales mit den Blendläden in der Villa, erkennen. Während die Vögel mit ihren langen Schwänzen und den Pfauenaugen dort der Natur abgeschaut sind, erscheinen sie hier mit ihrem metallisch schimmernden Gefieder und der impressionistischen Malweise gänzlich denaturiert. Möglicherweise wäre die richtige Bezeichnung dieses Stiles, dieser Ästhetik der Verkehrung, Stil der Perversion.

\section{Literatur:}

De Franciscis, A., La Villa Romana di Oplontis, Recklinghausen 1975.

Andreae, B. / Kyrieleis, H. (Hgg.), Neue Forschungen in Pompeji, Recklinghausen 1975.

JASHEMSKI, W. F., The Gardens of Pompei, vol. II, New Rochelles 1993.

ANDREAE, B. „Rus in urbe“, in: Bertelli, C. (Hrsg.), La Città gioiosa, Milano 1996, 53 108, besonders 57-59, Abb. 47-48. 


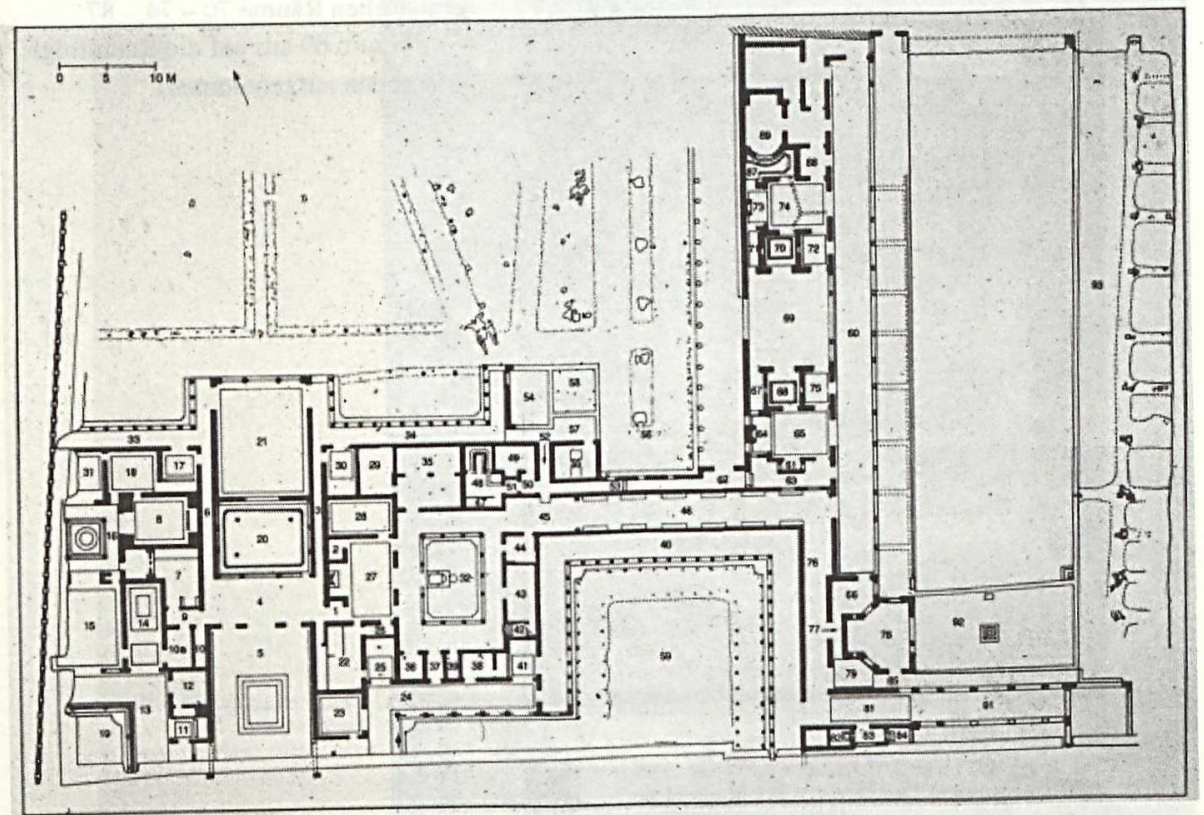

Abbildung 1:

Grundriß der Villa von Oplontis (Torre Anunziata) mit dem neronischen Erweiterungstrakt im Osten. Fünfeckiger Audienzsaal: Raum 78; Wartehalle: Raum 46 


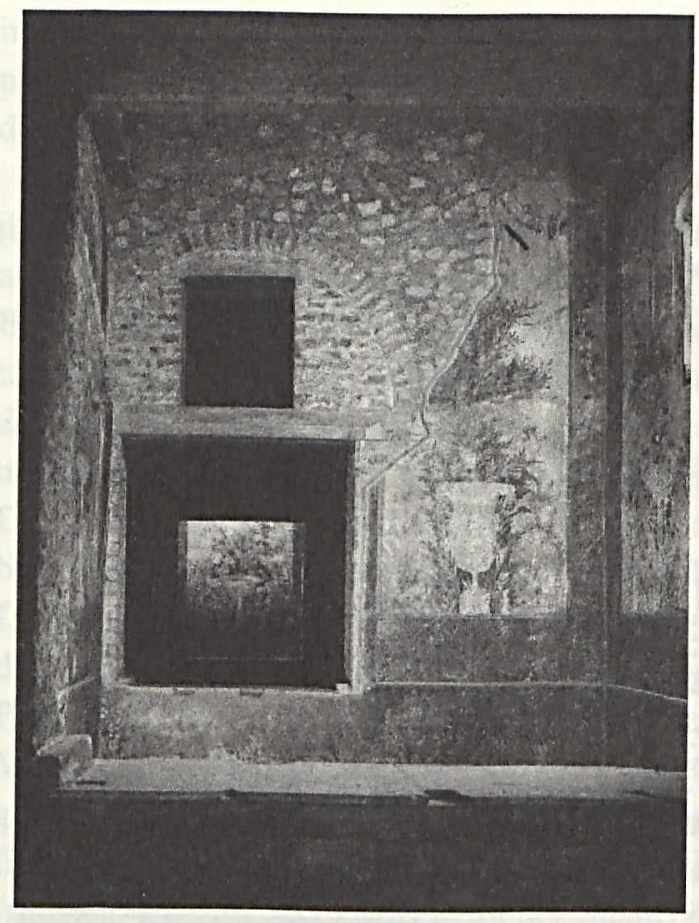

Abbildung 2:

Durchblick durch die hintereinander gestaffelten Räume 70 - 74-87 (von Raum 69 aus auf die Raumfolge im Norden aufgenommen)

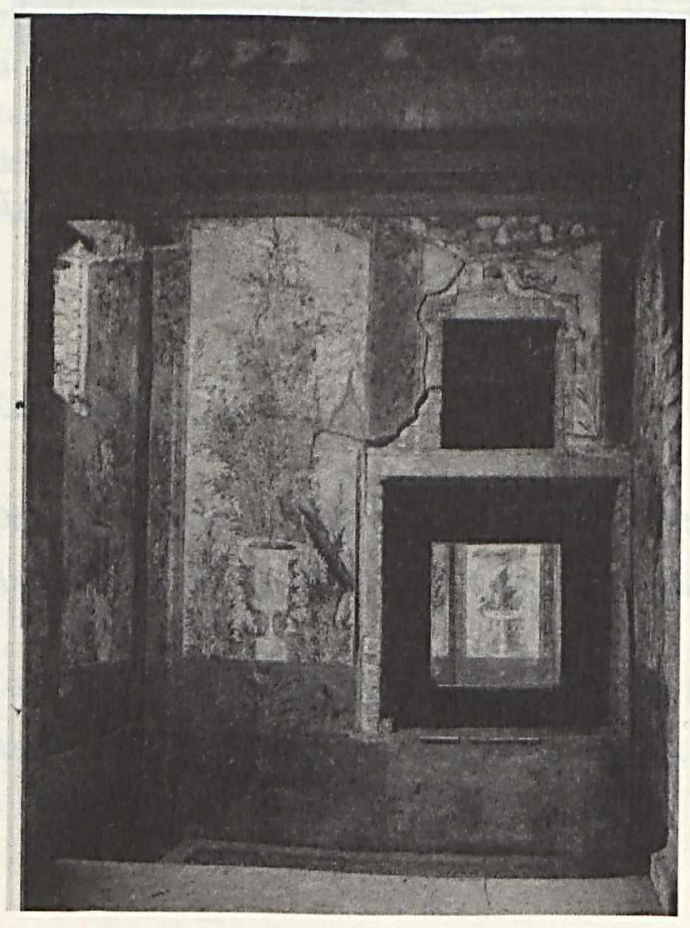

Abbildung 3:

Durchblick durch die hintereinander gestaffelten Räume 68 - 74-87 (von Raum 69 aus auf die Raumfolge im Süden aufgenommen) 


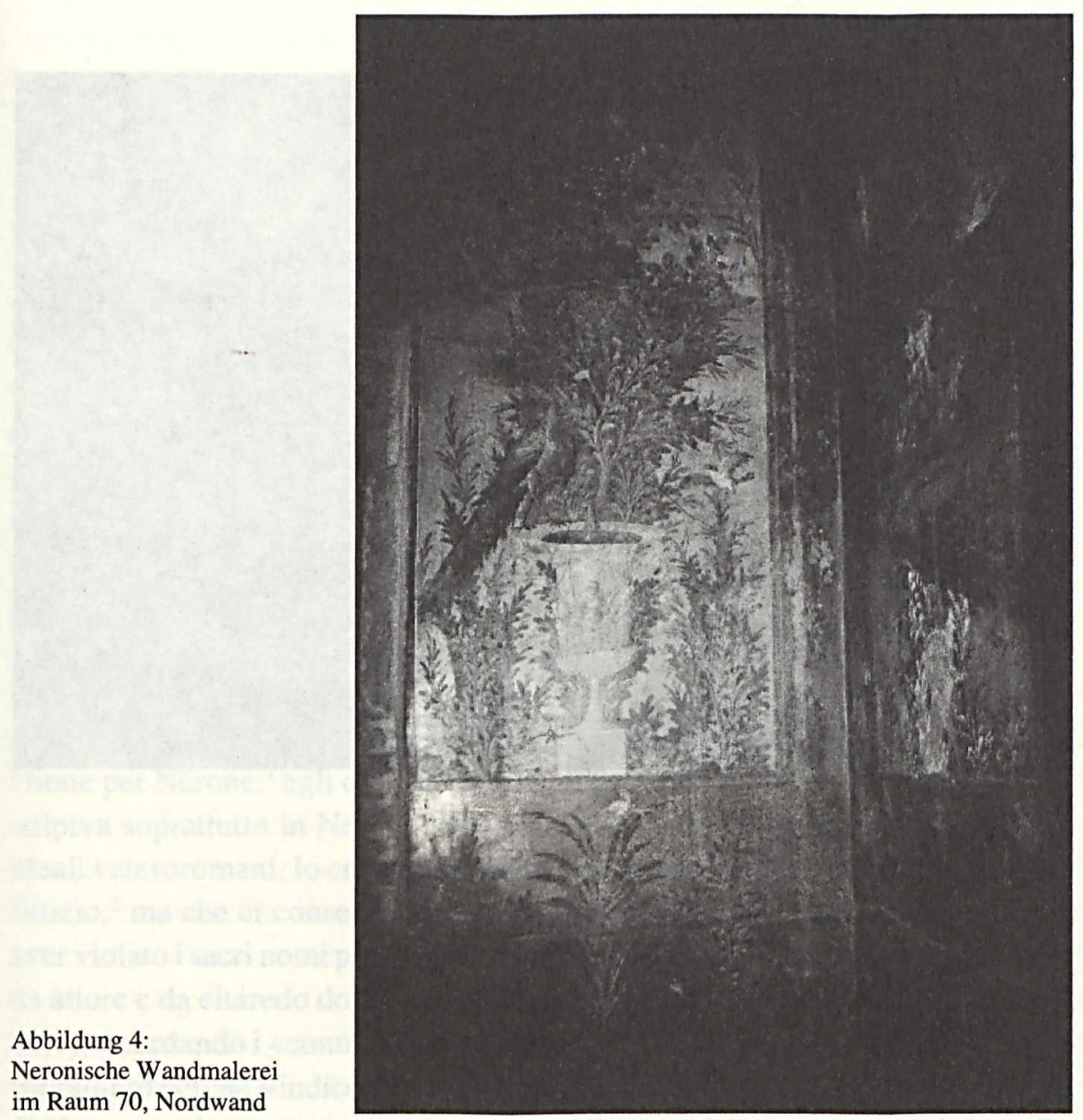




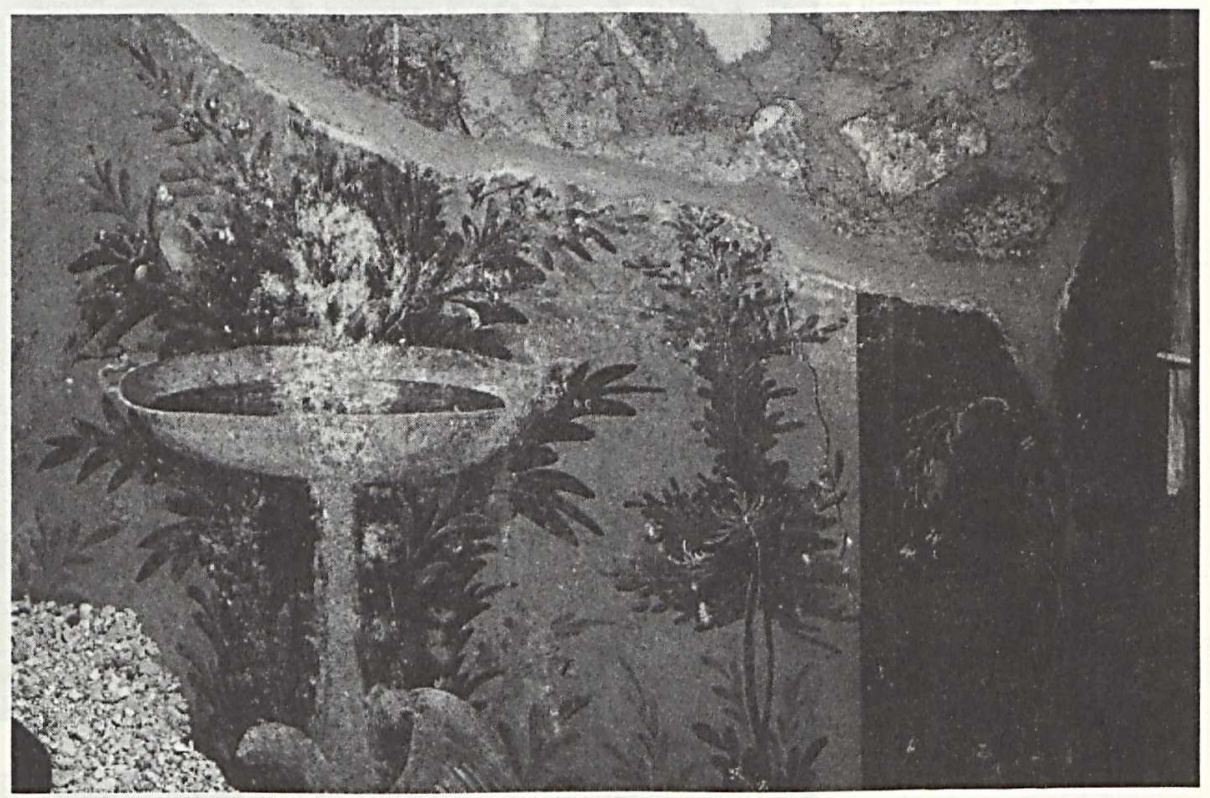

Abbildung 5:

Dasselbe, Westwand (Aufnahme während der Ausgrabung).

Alle Aufnahmen vom Verfasser 